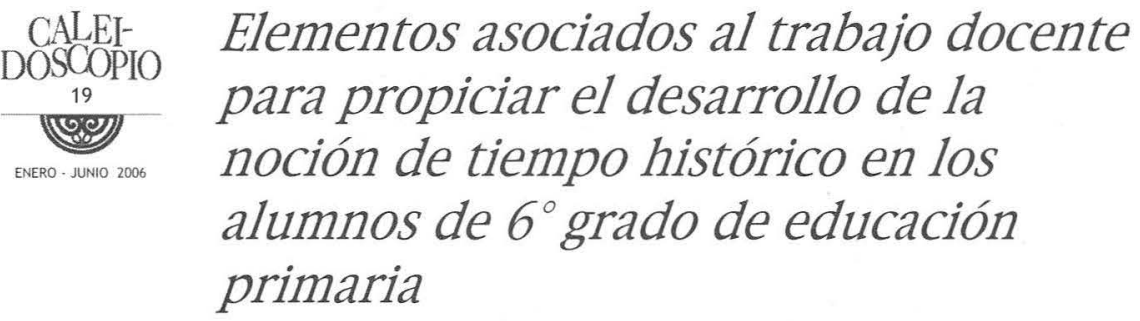

ANTONIO ORTIZ SANDOVAL

Unidad 011 Universidad Pedagógica Nacional

DESARROLLO DE LA NOCIÓN DE TIEMPO HISTÓRICO: UN PROBLEMA EN EL TRABAJO DOCENTE

Particularmente sobre el proceso de enseñanza - aprendizaje de la historia, la opinión de diversos actores del hecho educativo y el resultado de algunas evaluaciones señalan que "la cultura histórica de los estudiantes y egresados de educación básica en las generaciones recientes es deficiente y escasa"'.

Lo anterior plantea un grave problema que tiene que subsanarse, ya que "el ejercicio de la reflexión histórica desarrolla capacidades que pueden transferirse hacia el análisis de la vida social contemporánea"2. De ahí que los programas de estudio vigentes de educación primaria separan las llamadas Ciencias Sociales en las asignaturas de Historia, Geografía y Educación Cívica, dándoles un carácter básico en la formación del niño mexicano.

El proceso de aprendizaje de la asignatura de Historia representa para los alumnos, la experiencia adquirida en el trabajo con éstos y con profesores de educación primaria, dos problemas:

Secretaría de Educación Pública. Educación básica. Primaria. Planes y Programas de Estudio 1993.. SEP, México, 1993, p. 91

2 Ibid., p. 92 
- Dificultad de comprensión, ya que poco se ha investigado en nues tro país, acerca de las habilidades o capacidades que los niños deben desarrollar para construir su conocimiento histórico, puesto que no sólo en la escuela el alumno aprende estos conocimientos, sino que también fuera de ella recibe una gran cantidad de información que conforman un cierto aprendizaje de la historia.

- Las insuficiencias del verbalismo y de la memorización como recursos básicos utilizados por lo docentes en el proceso de aprendizaje de la historia.

Tradicionalmente en el proceso de aprendizaje de la Historia, las fechas ocupaban un lugar central en los contenidos que el alumno debía memorizar, dado que los hechos históricos necesariamente se tienen que ubicar en un momento determinado, por lo que el Tiempo Histórico (TH) juega un papel preponderante en dicho proceso. $^{3}$

Por ello en los programas vigentes de educación primaria se establece que la noción de $\mathrm{TH}$, para los alumnos de este nivel educativo, tiene un sentido muy vago "por eso es fundamental atender con cuidado el desarrollo de esta noción en los niños" ${ }^{4}$. Desde el punto de vista didáctico, expresan que el TH ha tenido una importancia decisiva, tanto como el hilo conductor del curriculum, como un aspecto más del contenido del mismo.

El TH consiste en un sistema complejo compuesto de diversos subsistemas, en donde cada uno de ellos tiene una existencia en sí mismos y que se constituyen con sus propios desarrollos particulares. Lo que plantea la importancia de propiciar la construcción de esta noción, que se concibe como "la culminación de un desarrollo que comienza con la adquisición de las nociones de tiempo personal, continúa con el tiempo físico, y el tiempo psicológico para que a través del tiempo social llegar al tiempo histórico" ${ }^{n 5}$.

3 M. Carretero, M. Asensio. y J. L.Pozo. (comps.). La enseñanza de las ciencias sociales. Madrid, Narcea, 1989.

4 Secretaría de Educación Pública. Libro para el maestro. Historia sexto grado. SEP, México, 1994, p. 22.

Ibid., p. 133. 
La SEP6 expresa que durante mucho tiempo se pensó que la dificultad en el aprendizaje de la historia radicaba en las formas de transmisión de los contenidos de historia, por lo que estableció la implementación de técnicas de enseñanza apropiadas, sin embargo se considera que conjuntamente con las formas de enseñar se encuentra el paradigma desde el cual el profesor concibe a la historia, que se manifiesta en el énfasis que él le da al presentar los hechos históricos y utilizando diversas maneras para ello.

Lo que implica que el estudio de la historia no es una finalidad en sí misma con su larga lista de fechas, personajes y acontecimientos, sino que por el contrario se debe de propiciar en el niño el desarrollo de sus capacidades y aptitudes que le lleven a dominar estrategias de estudio. Como lo afirma Luc "Lo importante no es sólo lograr la adquisición de conocimientos, sino hacer aprender el proceso cognitivo".

\section{EL PROBLEMA}

La problemática a investigar se refiere a la descripción y comprensión de algunos de los elementos asociados al trabajo docente en el desarrollo de la noción de $\mathrm{TH}$, tal y como lo plantea el programa de esta asignatura en educación primaria que expresa, "Al iniciar el estudio histórico sistemático, un elemento constante de la enseñanza será promover la adquisición progresiva de esquemas de ordenamiento histórico en grandes épocas"8 este planteamiento permite al docente el diseño de estrategias didácticas orientadas en este sentido.

Si se considera que el proceso de enseñanza - aprendizaje, es el punto específico que realizan los docentes y alumnos, éstos son el punto focal de esta investigación, particularmente en el $6^{\circ}$ grado de educación primaria puesto que en este grado tanto alumnos como docentes han tenido aproximaciones más formales con la Historia

Secretaría de Educación Pública, Ibid.

Ibid., p. 38.

8 Secretaría de Educación Pública., op. cit., p. 92. 
como asignatura, lo que implica por parte de los profesores un trabajo docente más sistematizado en torno a la misma y por los alumnos una mayor evolución en el desarrollo de la noción de TH.

Por lo tanto el objetivo de esta investigación es el de describir y comprender la concepción de Historia de las profesoras, la importancia que le otorgan a esta asignatura en la formación de los alumnos y las formas de enseñarla, como elementos asociados al trabajo docente que propicie el desarrollo de la noción de tiempo histórico (TH) reflejado en los niveles de conceptualización logrados por los alumnos de sexto grado de educación primaria.

La investigación gira en torno a la siguiente interrogante: ¿La forma de enseñar la Historia, la concepción que los profesores tiene sobre esta disciplina y la importancia otorgada por los mismos a esta asignatura en la formación de los alumnos como elementos que están asociados a la práctica docente permiten propiciar el desarrollo de la noción de tiempo histórico en los alumnos de sexto grado de educación primaria de escuelas urbanas del municipio de Aguascalientes?

De donde se desprenden algunas preguntas particulares que se trataron de ir despejando en el desarrollo de la investigación:

¿Qué concepción de Historia poseen los docentes?

¿Qué importancia tiene para el docente esta asignatura en la formación del alumno de educación primaria?

¿Cuáles son las formas de enseñar la Historia que utilizan los docentes? ¿Cuáles son los niveles de conceptualización del desarrollo de la noción de $\mathrm{TH}$ en los alumnos de sexto grado de educación primaria?

\section{¿CÓMO ABORDAR ESTE PROBLEMA?}

Se llevó a cabo con cinco docentes de tres escuelas primarias urbanas de la ciudad de Aguascalientes, las cuales fueron designadas por criterios socioeconómicos de Gelles y Levine9 que son: nivel de educación, ocupación o tipo de trabajo y lugar de residencia. Por lo tanto

9 Gelles R y Levine A. Introducción a la Sociología. Con aplicaciones a los países de habla hispana. Mc Graw Hill, México, 1996. 
se seleccionaron a partir del nivel socioeconómico que las caracteriza en su entorno. Nivel socioeconómico bajo(escuela 3), ubicada en una colonia urbana marginada, donde los padres de familia en su mayoría se dedican al comercio informal, a algunos oficios como albañiles o herreros y jornaleros, con ingresos mínimos y con un nivel educativo que apenas alcanza la educación primaria. Nivel socioeconómico medio (escuela 2), ubicada en un fraccionamiento de clase media, donde los padres de familia son profesionistas o se dedican a actividades de servicio y cuyos niveles de escolarización son o de nivel superior o de educación media. Nivel socioeconómico alto (escuela 1), ubicada en un fraccionamiento residencial, cuyos padres de familia son empresarios o tienen un negocio propio, así como docentes de la UAA y sus niveles de escolarización van desde el nivel medio hasta el posgrado.

Las tres escuelas tienen un total de cinco grupos de $6^{\circ}$ grado y por consiguiente cinco profesoras, que en el desarrollo de este trabajo se identifican con base en la escuela y el grupo que atendían (Maestra 1A, 1B, $2 \mathrm{~A}, 2 \mathrm{~B}$ y $3 \mathrm{~A}$ ) ésta última sólo se identifica con la letra A porque el grupo era único y los alumnos de estos grupos, se identifican por el número de la escuela, la letra del grupo (A o B) y el número de lista. La caracterización de los sujetos se presenta a continuación en el cuadro $\mathrm{N}^{\circ} 1$ :

Cuadro 1. Caracterización de LAS PROFESORAS INVESTIGADAS DISEÑO DE LA INVESTIGACIÓN

\begin{tabular}{|c|c|c|c|c|c|}
\hline $\begin{array}{l}\text { Indica- } \\
\text { dor }\end{array}$ & EDAD & $\begin{array}{l}\text { AÑOS } \\
\text { DE } \\
\text { SER- } \\
\text { VICIO }\end{array}$ & NORMAL DE EGRESO & $\begin{array}{l}\text { PERÍODO } \\
\text { DE ESTU- } \\
\text { DIOS }\end{array}$ & ESTUDIOS POSTERIORES \\
\hline $1 \mathrm{~A}$ & $\begin{array}{c}36 \\
\text { años }\end{array}$ & 17 & Normal del Estado de Ags. & $\begin{array}{r}1978- \\
1982\end{array}$ & $\begin{array}{l}\text { Normal Sup. Matemáticas } \\
\text { UPN Lic. en Educ. Básica } \\
\text { Plan } 1979 \text { (titulada) }\end{array}$ \\
\hline 1B & $\begin{array}{c}39 \\
\text { años }\end{array}$ & 22 & Colegio Guadalupe Victoria & $\begin{array}{l}1978- \\
1982\end{array}$ & $\begin{array}{l}\text { UPN } 5^{\circ} \text { semestre de la Li- } \\
\text { cenciatura en Educación } \\
\text { Plan } 1994 \\
\text { UAA 3er semestre de Lic. } \\
\text { Investigación Educativa }\end{array}$ \\
\hline $2 \mathrm{~A}$ & $\begin{array}{c}52 \\
\text { años }\end{array}$ & 23 & Normal del Estado de Jal. & $\begin{array}{l}1968- \\
1971\end{array}$ & $\begin{array}{l}\text { Normal Sup. } \\
\text { C. Sociales }\end{array}$ \\
\hline $2 \mathrm{~B}$ & $\begin{array}{c}42 \\
\text { años }\end{array}$ & 24 & $\begin{array}{l}\text { Centro Regional de Educación } \\
\text { Normal de Ags. }\end{array}$ & $\begin{array}{l}1972- \\
1976\end{array}$ & $\begin{array}{l}\text { Normal Sup. Español (ti- } \\
\text { tulada) } \\
\text { UPN Lic en Edu-cación Pri- } \\
\text { maria Plan } 1985 \text { (titulada) }\end{array}$ \\
\hline $3 \mathrm{~A}$ & $\begin{array}{c}35 \\
\text { años }\end{array}$ & 16 & $\begin{array}{l}\text { Normal Rural Justo Sierra Mén- } \\
\text { dez de Cañada Honda, Ags. }\end{array}$ & $\begin{array}{l}1980- \\
1984\end{array}$ & Nor. Superior Español \\
\hline
\end{tabular}

$\begin{array}{lllllllllllllll}C & A & L & E & I & D & O & S & C & O & P & 1 & O & & \end{array}$


Es descriptiva cualitativa tomando como base el planteamiento de Briones ${ }^{10}$ que plantea a la descripción como la representación de personas o cosas por medio del lenguaje, refiriendo o explicando sus distintas partes, cualidades o circunstancias.

Para Tamayo" "la investigación descriptiva comprende la descripción, registro, análisis e interpretación de la naturaleza actual, y la composición y los procesos de los fenómenos...la investigación descriptiva trabaja sobre realidades de hecho y su característica fundamental es la de presentarnos una interpretación correcta". Asimismo los "estudios descriptivos buscan especificar las propiedades importantes de personas, grupos, comunidades o cualquier otro fenómeno que sea sometido a análisis" ${ }^{12}$. Por ello es importante establecer que esta investigación describe, identifica y trata de comprender los elementos que están asociados al trabajo docente de las profesoras investigadas, así como el nivel de conceptualización de la noción de TH que tienen los alumnos de dichas docentes en el momento en el que se les aplicó el instrumento.

\section{CATEgorías teóRICAs}

Se pueden identificar dos, una es los elementos que están asociados al trabajo docente, y que en este caso son: formas de enseñar la Historia, concepción de Historia y la importancia que le otorga a esta asignatura en la formación del alumno. La otra referida a los alumnos es el desarrollo de la noción de tiempo histórico, en donde se establecen tres niveles de conceptualización para su desarrollo que son orden de acontecimientos dentro de una secuencia (cronología), agrupamiento de acontecimientos concurrentes en el tiempo (simultaneidad) y establecer un sentido de continuidad entre el pasado y el presente (continuidad).

10 Recuperado del Diccionario de la Lengua Española por G. Briones Métodos y técnicas avanzadas en investigación aplicadas a la Educación y a las Ciencias Sociales. Módulo 1. Epistemología y metodología de la investigación social. Santiago de Chile, Programa interdisciplinario de Investigaciones en Educación, 1996.

11 Tamayo y Tamayo, El proceso de la investigación científica, México, Limusa, , 1996, p. 54.

12 R. Hernández Sampieri, Metodologá de la investigación, Colombia, Mc Graw Hill, 1991, p. 60 . 
Las utilizadas en esta investigación son las que permitieron hacer una descripción de la realidad estudiada para lograr el acercamiento al objeto de estudio, a continuación se mencionan.

Entrevista semiestructurada abierta, según Del Rincón 13 "aporta a la metodología constructivista la profundidad y las perspectivas de los entrevistados, permitiendo la interpretación de los significados de las acciones." Se les cuestionó a las docentes acerca de sus concepciones sobre la Historia y la importancia que le otorgan a esta disciplina en la formación de sus alumnos, además de una serie de datos generales acerca de su formación y experiencia profesional a efecto de caracterizarlas.

Observación en el aula, se llevó a cabo por medio de una lista de verificación, en donde como lo plantea Stallings y Mohalman compilados por UPN ${ }^{14}$, "se prepara una lista de conductas supuestas y cada vez que tiene lugar este comportamiento, durante la unidad de tiempo de observación especificada, el observador pone una señal junto a la categoría correspondiente." Para lo cual esta identificación de la forma de enseñar la Historia, se hizo con base en la caracterización de Aebli ${ }^{15}$ y tomando como referencia las identificadas por Valencia ${ }^{16}$ en cuyos resultados de su investigación ha obtenido. Asimismo se realizó una observación ampliada, con el propósito de complementar lo que sucedió en la clase.

Encuesta, consistió en una prueba objetiva, que según Del Rincón ${ }^{17}$ se consideran como los instrumentos que mejor contrarrestan la subjetividad del profesor, ya que exigen respuestas concretas y evitan la preocupación del alumno por la redacción y presentación.

13 D. Del Rincón., I. Arnal, A. Latorre y A. Sans, Técnicas de investigación en las Ciencias Sociales, Madrid, Dykinson, 1995, p. 308.

14 Universidad Pedagógica Nacional. Diseño de la investigación educativa. Antología. Unidad UPN 011 . Aguascalientes, 1999, p. 116. comp.. Mtra. Josefina Mercado Haro.

15 Ibid.

16 A. Valencia García, Las formas de enseñar Ciencias Sociales: el caso de la Historia en profesores de educación primaria. Tesis para obtener el grado de Maestría UAA, Aguascalientes, 1998.
C
A L E
D $O$
S
C $\mathrm{O}$
P 10 
La idea de aplicar este instrumento, surge por la necesidad de identificar el nivel de conceptualización que los alumnos tienen con respecto a la noción de TH, lo que permitirá tener un punto de referencia para establecer la relación con los elementos asociados al trabajo docente del profesor que facilita o no su desarrollo.

La primera parte del instrumento corresponde a los datos generales del alumno, la segunda contiene los ítems a partir de los cuales se pretende que los alumnos evidencien los niveles de conceptualización de la noción de $\mathrm{TH}$.

Ítems I, II y III se refieren a la cronología, o sea al nivel de ordenamiento de acontecimientos dentro de una secuencia, con un total de 17 respuestas, lo que implica que si contesta correctamente 13 o más de éstas, entonces parece ser que se ha logrado alcanzar este nivel, en cambio si contesta correctamente de 8 a 12 entonces se encuentra en un nivel medio y si acierta en 7 o menos se ubica en un nivel bajo.

Ítems IV, V y VI se refieren a la simultaneidad, es decir al nivel de agrupamiento de acontecimientos concurrentes en un mismo momento, con un total de 13 respuestas, si contesta 10 o más correctamente, entonces está en un nivel alto, si contesta correctamente de 6 a 9 respuestas se encuentra en nivel medio de desarrollo y si acierta 5 o menos se ubica en un nivel bajo.

Ítems VII y VIII se refieren a la continuidad es decir la relación entre presente - pasado, con 7 respuestas, si responde correctamente a las 7 se encuentra en un nivel alto, si contesta correctamente de 5 a 6 , se ubica en un nivel medio de desarrollo y si acierta 4 o menos el nivel es bajo.

CONCEPCIÓN DE HiSTORIA DE LOS DOCENTES DE EDUCACIÓN PRIMARIA: ¿UNA NUEVA HISTORIA?

Actualmente se plantea la concepción de una Nueva Historia, en donde Burke $^{18}$ establece una serie de comparaciones entre ésta y la historia tradicional, tratando de describir las características tendientes a que la nueva historia sea una historia total. Dichas comparaciones son:

17 Ibid. 
- La nueva historia es una reacción contra el paradigma tradicional en donde el objeto de estudio era la política, mientras que la nueva historia se interesa por toda la actividad humana, es decir se parte de la premisa de que todo tiene su historia.

- En la historia tradicional se piensa a ésta como una narración de acontecimientos, mientras que en la nueva se utiliza más el análisis de estructuras.

- El paradigma tradicional presenta una visión de los acontecimientos desde arriba, centrado en el estudio de los grandes personajes, las grandes hazañas, etc., mientras que la otra, estaría en una historia desde abajo, o sea las opiniones de la gente común y corriente.

- La historia tradicional establece que su base está en los documentos, a los cuales varios historiadores han cuestionado, porque presentan una sola visión de los hechos, que no siempre es la más cercana a la realidad; la historia desde abajo ha echado por tierra esta postura y se buscan otro tipo de evidencias, como, la tradición oral, las estadísticas, etc.

- En el paradigma tradicional las explicaciones históricas a menudo no consiguen dar respuestas razonables a todos los cuestionamientos que surgen de los movimientos históricos, en cambio la nueva historia plantea respuestas multifactoriales a los acontecimientos históricos. "El movimiento de la historia desde abajo refleja también una nueva decisión de adoptar los puntos de vista de la gente corriente sobre su propio pasado con más seriedad que la acostumbran los historiadores profesionales. Lo mismo vale para algunas formas de historia oral." ${ }^{\prime \prime}$; lo que significa que para esta concepción los sujetos ordinarios tienen y hacen Historia y no es exclusiva de los grandes personajes de la misma. Esta concepción es la que predomina en el programa de Historia de educación primaria, ya que se pretende analizar los sucesos referidos al pensamiento, a los avances científicos y tecnológicos, a las manifestaciones artísticas y en general a la vida cotidiana de los individuos; lo que conlleva a plantear que todos los sujetos hacen historia.

18 P. Burke, Formas de hacer Historia. Alianza, Madrid, 1993.

$\begin{array}{lllllllllllll}C & A & L & E & \text { I } & D & O & S & C & O & P & \text { I } & O\end{array}$ 
El reto para la nueva historia y para los docentes inmersos en esta problemática es como relacionar los grandes sucesos con la vida cotidiana, por lo que al contrario de esta concepción las profesoras entrevistadas confirman que la historia es estudio del pasado, como se puede apreciar en las siguientes respuestas:

Maestra (2B). ¿para ud. qué es la historia? "Para mí, la historia es el estudio de hechos pasados, ¿si? Puede ser desde la historia personal, la historia familiar y pues ya la historia de un estado, hasta de una nación y del mundo. Hechos pasados." Con base en lo expresado por esta maestra, esta asignatura es el estudio del pasado, lo que significa que en el proceso de enseñanza - aprendizaje, lo que revisan de los contenidos de esta materia es con una visión de hechos que ya sucedieron, por lo que para los alumnos son aprendizajes carentes de significatividad.

Maestra (2A) responde "la fistoria... bueno, yo pienso que son los sucesos más relevantes que ha tenido nuestra... nuestra, ¿cómo le quisiera decir? nuestro país." Con esta concepción para esta maestra esta materia sigue siendo el estudio de acontecimientos pasados, adicionando que deben ser aquellos que han sido relevantes con lo que se mantiene la idea de que los sucesos con esa característica son los de carácter político y militar, dejando de lado la cotidianeidad de los sujetos que conforman los grupos sociales y por ende no se ajusta a la concepción de la nueva Historia que toma como elementos relevantes los referidos a los hechos cotidianos de los sujetos y su impacto en los cambios sociohistóricos, por lo que no se forma en los alumnos un pensamiento histórico.

Del mismo modo la maestra (1 A) dice "pues es la ciencia que se dedica al estudio del pasado y de cómo han ido cambiado las sociedades, los pueblos, qué cambios han experimentado a través del tiempo." En esta concepción se mantiene la idea del pasado, asimismo se puede apreciar que para esta maestra el cambio en el tiempo es fundamental para el estudio de esta disciplina, sin embargo, lo expresado por esta profesora parece ser que no implica una reflexión sobre dichos cambios, más bien es sólo información y/o descripción de ellos para los alumnos.

19 Ibid., p. 19 
Estas concepciones sobre la disciplina de la historia expresada por las maestras, en el fondo plantea rehuir el compromiso social con el presente, que trata de romper de modo artificial la línea temporal que es continua, como lo afirma Aguirre Rojas ${ }^{20 " ~ c u a l q u i e r ~ p r e s e n t e ~}-\mathrm{y}$, por ende cualquier pasado - no es mas que una compleja articulación estratificada de distintos pasados todavía presentes".

Se puede apreciar a través de estas respuestas de sus concepcio nes acerca de la Historia que están orientadas al estudio del pasado matizadas por algunos destellos de otras concepciones, como en el caso de la maestra (1B) que respondió: "La historia, es lo que el hombre ha hecho... y que nos sirve como referente en el presente." En esta respuesta continua la concepción del pasado, sólo que lo utiliza como referente para el otro elemento que incluye que es el presente, sin embargo parece ser que no se concibe a los sujetos como hacedores de su propia historia lo que de alguna manera conduce a que los alumnos aprendan los contenidos de Historia únicamente como información pero sin reflexionar que nuestro presente es producto de ese pasado y que en nuestras manos está el construirlo de manera diferente.

También la maestra (3A) afirma: "bueno, pues para mi la historia es una disciplina que habla sobre los cambios del pasado y actuales también, en nuestra sociedad, y las manifestaciones que lleva a efecto el hombre y que interactúa con otras ciencias, con otras disciplinas." En esta respuesta hay dos elementos, uno relacionado con los cambios que estudia la Historia, tanto del pasado como del presente, lo que significa que permite la formación de sujetos que reflexionen sobre dichos cambios, el otro es la relación con otras áreas del conocimiento lo que significa que los acontecimientos que se estudien tienen que analizarse desde diferentes disciplinas de lo social; pero no se enfatiza la reflexión sobre dichos acontecimientos que permitan formar un pensamiento histórico.

Estas concepciones que las maestras entrevistadas expresaron permean su hacer educativo en el proceso de enseñanza de esta asignatura, presentando el conocimiento histórico como hechos pa-

20 C. A.Aguirre Rojas, Antimanual del mal historiador o como hacer una buena historia crítica, México, La Vasija, 2003, p. 21. 
sados que no tienen ninguna repercusión en la situación actual que vive el alumno, por consiguiente no se propicia la formación de un sujeto histórico, con base en el desarrollo de las diferentes nociones necesarias para ello.

IMPORTANCIA OTORGADA POR LAS MAESTRAS A LA ASIGNATURA DE HISTORIA EN LA FORMACIÓN DEL ALUMNO DE EDUCACIÓN PRIMARIA

El programa de historia plantea que "esta disciplina tiene un especial valor formativo, no sólo como elemento cultural que favorece la organización de otros conocimientos, sino que también como factor que contribuye ala adquisición de valores éticos personales y de convivencia social y a la afirmación consciente y madura de la identidad nacional". ${ }^{21}$

En este sentido se puede reconocer en lo que la maestra $\mathbf{2 A}$, expresa sobre la importancia de esta asignatura que estriba en la formación de valores patrios que implican el reconocimiento a nuestros héroes: Yo pienso que sí. Porque al niño, primeramente le enseñamos a, éste, el amor a la Patria, ¿verdad? después le enseñamos a tener valores, y viene también, el valor a nuestros símbolos patrios, el valor a nuestros... el reconocimiento más bien a nuestros héroes, nuestra nacionalidad, nuestra soberanía.

La maestra 3A, plantea que a partir del conocimiento de esta asignatura los alumnos pueden ubicarse en el momento que viven y como pueden formar parte de la misma, lo que manifiesta una asunción de su importancia en la formación de los niños: Sí, es muy importante que se enseñe, porque a través de la historia los niños pueden conocer los grandes procesos por los que ha pasado la humanidad y que ellos se sitúen y se ubiquen, ellos como parte de esa historia porque posteriormente ellos pasarán a formar parte de la misma. Parte de su ubicación espacial...

Para la maestra 1B, la importancia de la historia se encuentra en la formación de la identidad, al manifestar: Sí, si no conoces historia, se niega a sí mismo y a su desarrollo. Finalmente, yo les digo a los niños, bueno, si no conocen su historia, se están negando ustedes y no saben identificar como

21 Ibid, p. 89 
mexicanos no van a tener identidad nacional, que a lo mejor, si sería un discurso pedagógico, si Ud. quiere, pero en realidad, es una realidad y sí estoy de acuerdo en que deben de tener un sentimiento de identidad nacional, que conozcan su historia.

La maestra 1A afirma que su importancia está en los hechos del pasado que han conformado lo que somos ahora y por consiguiente no otorga a los sujetos actuales el hacer historia: Bueno, porque el momento que estamos viviendo, es producto de lo que ha pasado anteriormente, todos esos acontecimientos y todos esos hechos importantes, que han determinado hasta qué punto somos ahora.

Para la maestra 2B, esta asignatura no es tan importante en la medida en que los contenidos curriculares son bastantes y por consiguiente exige mucho del docente afirmando que lo esencial en la Historia es el aprendizaje de los alumnos y la realidad que viven: Sí, si es importante pero...no al grado que se nos exige, o sea, el programa tan extenso, como que a mí me gustaría que nada más fueran como nociones, como para que el niño se ubicara en una realidad y que a partir de esa pues también ha habido cosas que han ocurrido antes de, y que lo que ocurra después, se vuelve historia. ¿verdad? pero nada más, como una noción.

Las opiniones de las maestras entrevistadas con respecto a la importancia que tiene el enseñar la Historia en la formación de los alumnos, se nota como permea la concepción que tienen de la disciplina como hechos del pasado. Persiste un discurso que avala la importancia pero en el hacer se manifiesta la falta de relevancia para la enseñanza de la Historia y por ende una falta de formación de alumnos con un pensamiento histórico, al no propiciar el nivel de conceptualización de continuidad, es decir, la relación presente pasado, fundamental para la construcción y desarrollo de la noción de tiempo histórico (TH) que permita la formación de individuos históricos, en donde asumen la facultad de mejorar sus condiciones de vida social.

FORMAS DE ENSEÑAR LA HISTORIA EN $6^{\circ}$ GRADO DE EDUCACIÓN PRIMARIA

La enseñanza desde la perspectiva constructivista establece que las estrategias utilizadas se modifican para considerar al sujeto un ente 
activo en su propia formación, por lo tanto el docente representa, según esta concepción, un ente facilitador de procesos, en donde su intervención debe regirse por el principio de la ayuda pedagógica a los alumnos que más lo requieran, en la construcción de sus aprendizajes.

Sin embargo Las prácticas educativas en educación primaria en torno al proceso enseñanza aprendizaje de la historia, siguen privilegiando un aprendizaje memorístico, mecánico y plagado de informaciones con fechas y nombres, los cuales presentan a los niños una historia estática y cuyos hechos son protagonizados exclusivamente por los grandes personajes de la misma. Valencia ${ }^{22}$ concluyó en su investigación realizada con profesores de primaria de la ciudad de Aguascalientes que de las doce formas de enseñar, planteadas por Aebli ${ }^{23}$, en la enseñanza de la historia en educación primaria, los docentes utilizan leer con los alumnos y escribir y redactar textos, como formas importantes de construir conocimientos, sin embargo la manera tan rutinaria como se usan llegan a perder su eficacia. Otras formas que también utilizan son las de contemplar y observar y ejercitar y repetir y aplicar. Esta conclusión es la referencia para identificar las formas de enseñar de las maestras de este estudio.

La identificación de las formas de enseñar, se hizo por medio de observación en el aula, a cinco docentes, durante un período de cuatro meses, de la clase de Historia, por medio de una lista de verificación, que se complementó con un registro abierto de observación de las interacciones maestra (Ma.) -alumno(a) (Ao., Aa.) y alumno(a)-alumno(a). Se presentan las formas de enseñar identificadas en el cuadro $\mathrm{N}^{\circ} 2$ :

\footnotetext{
22 A. Valencia García, op cit.

23 H. Aebli, op. cit.
} 


\section{CuAdRo 2. Formas dE ENSEÑAR IDENTIFICADAS}

\begin{tabular}{|c|c|c|}
\hline $\begin{array}{l}\text { MAES : } \\
\text { TRA }\end{array}$ & $\begin{array}{l}\text { FORMAS DE } \\
\text { ENSEÑAR }\end{array}$ & ACCIONES \\
\hline \multirow{3}{*}{$1 \mathrm{~A}$} & $\begin{array}{l}\text { Leer con los } \\
\text { alumnos }\end{array}$ & $\begin{array}{l}\text { Lectura de los alumnos en silencio. La Ma. les dice a los Aos. que } \\
\text { lean determinados fragmentos del texto. }\end{array}$ \\
\hline & Ejercitar y repetir & $\begin{array}{l}\text { Preguntas y respuestas orales. Pregunta para recordar, sobre acti- } \\
\text { vidades anteriores, una película que aborda lo de la Revolución y } \\
\text { una obra de teatro sobre la misma temática. } \\
\text { Explicación de los Aos. de un trabajo que elaboraron en equipo. }\end{array}$ \\
\hline & Elaborar & $\begin{array}{l}\text { Cada equipo pasa al frente a realizar la explicación sobre lo que } \\
\text { elaboraron, utilizando para ello pliegos de papel bond donde } \\
\text { escribieron lo que van a exponer. }\end{array}$ \\
\hline \multirow{3}{*}{ 1B } & $\begin{array}{l}\text { Leer con los } \\
\text { alumnos }\end{array}$ & $\begin{array}{l}\text { Lectura de los Aos. en voz alta, leyendo lo que la Ma. les indica y } \\
\text { a quien determina. }\end{array}$ \\
\hline & Ejercitar y repetir & $\begin{array}{l}\text { Preguntas orales, cada que un Ao. termina de leer la Ma pregunta } \\
\text { por el contenido de lo leído, algunos Aos contestan }\end{array}$ \\
\hline & Elaborar & $\begin{array}{l}\text { Convertir en noticia algunos de los contenidos del texto y pasan } \\
\text { a leerla al grupo. }\end{array}$ \\
\hline \multirow{3}{*}{$2 \mathrm{~A}$} & $\begin{array}{l}\text { Leer con los } \\
\text { alumnos }\end{array}$ & $\begin{array}{l}\text { Lectura de los Aos. en voz alta, la cual es guiada por la Ma., que } \\
\text { también indica quien debe ir leyendo, al término de la lectura } \\
\text { pregunta a los Aos. sobre el contenido del texto. }\end{array}$ \\
\hline & $\begin{array}{l}\text { Ejercitar y repetir } \\
\text { Narrar y referir }\end{array}$ & $\begin{array}{l}\text { Preguntas y respuestas orales } \\
\text { Ma. narra hechos históricos. }\end{array}$ \\
\hline & Elaborar & $\begin{array}{l}\text { Pasa un Ao. por equipo y lee lo que escribieron en la hoja y explica el } \\
\text { dibujo correspondiente, la Ma. va pegando cada uno de los trabajos } \\
\text { de los Aos. en el pizarrón. }\end{array}$ \\
\hline \multirow{3}{*}{$2 \mathrm{~B}$} & $\begin{array}{l}\text { Leer con los } \\
\text { alumnos }\end{array}$ & Lectura de la Ma. en voz alta \\
\hline & Elaborar & $\begin{array}{l}\text { Elaboración de un breve escrito por equipo y pasan a escribirlo en } \\
\text { un cuadro en el pizarrón }\end{array}$ \\
\hline & $\begin{array}{l}\text { Ejercitar y repetir } \\
\text { Narrar y referir }\end{array}$ & $\begin{array}{l}\text { Preguntas y respuestas orales } \\
\text { Ma. narra y explica hechos históricos }\end{array}$ \\
\hline \multirow{3}{*}{$3 \mathrm{~A}$} & $\begin{array}{l}\text { Leer con los } \\
\text { alumnos }\end{array}$ & $\begin{array}{l}\text { Lectura en silencio de los alumnos, para después hacer lectura } \\
\text { comentada por la Ma de cada párrafo }\end{array}$ \\
\hline & Ejercitar y repetir & Preguntas y respuestas orales \\
\hline & Elaborar & $\begin{array}{l}\text { Aos de cada equipo pasan a leer la pregunta y respuesta que } \\
\text { les tocó }\end{array}$ \\
\hline
\end{tabular}

Como se puede apreciar en este cuadro, leer con los alumnos, es una forma de enseñar que las cinco maestras observadas utilizan, en donde predomina la lectura de los alumnos ya sea en silencio o en voz alta y la lectura guiada y/o comentada por la maestra. Lo que refleja que es la más común y el alumno quede limitado a la indicación de la maestra sobre lo que tiene que leer, que en ocasiones no es de su interés y se presta a que los alumnos sólo simulen la lectura, por consiguiente la construcción de las nociones y del aprendizaje de los contenidos de historia no se propicia. Para sustentar esta afirmación 
se presenta como ejemplo el siguiente pasaje de esta forma de enseñar de una de las maestras observadas:

La maestra 3A, utiliza la lectura tanto en silencio como en voz alta y comentada, sin embargo la forma como plantea estas actividades, no manifiesta que se propicie un aprendizaje en los alumnos:

Ma. "Vamos a ver la clase de historia, saquen su libro de historia en la página 93 y vamos a leer en silencio hasta la página $95 . "$

La lectura en silencio de los Aos. dura unos 15 minutos, mientras la maestra recorre cada uno de los pasillos del aula cuidando de que todos estén leyendo.

Ma. Da por terminada la lectura y dice: " vamos ir leyendo párrafo por párrafo y les voy a explicar". Indica a un Ao. que inicie la lectura.

Ejercitar y repetir, esta es otra de las formas que es utilizada por las cinco maestras observadas, la única acción que se realiza es preguntas y respuestas orales, se orienta a que la maestra sea la única que cuestiona a los alumnos acerca del texto que leyeron o del tema que se aborda, que en la mayoría de las ocasiones hace caso omiso de las respuestas que los alumnos le dan, pero la falta de atención de las maestras al respecto genera que el alumno no logre obtener respuestas de lo que cuestiona.

Para la maestra 1A, el utilizar las preguntas orales es para recordar lo que se vio en la clase anterior, cuestiona en grupo y las respuestas de los alumnos a coro no permite darse cuenta del aprendizaje real de cada uno de ellos, además hace preguntas que no permiten un diálogo con los alumnos, tal y como se presenta en la siguiente descripción:

Ma. Pregunta sobre actividades anteriores una película que aborda lo de la Revolución y una obra de teatro sobre la misma temática: "¿Se acuerdan que la semana pasada vimos un película?"

Aos. "Sii" (a coro)

Ma. "¿De que trató?"

Ao. "De guerra"

Aa. "Sobre la forma de cómo los mexicanos nos peleamos"

Aa. "De la Revolución que hubo en México"

Ma. "¿Se acuerdan del viernes que fuimos al Teatro?"

Aos. "Sii" (a coro)

Ma. "¿Sobre que tema se trató la obra?"

Aos. "Sobre la Revolución"

Ma. Les indica que: "abran su libro de Historia en la pág. 109, el tema de la Revolución", y determina lo que cada equipo va a realizar. 
Narrar y referir, es otra de las formas de enseñar la historia que se presenta en dos de las cinco maestras observadas, en donde la Maestra narra hechos históricos, lo que se muestra a continuación es la manera en que es presentado el conocimiento a través de esta forma de enseñar. Para la maestra 2A, parece que es importante exaltar los valores patrios, de ahí que su narración gira en torno de este aspecto:

Ma. Comenta la importancia del patriotismo y dice: "amor a la tierra, a la patria es importante para luchar con valentía, a pesar de que los héroes como Villa y Zapata no sabían leer, pero sabían lo que era la patria, esto es ser mexicano, defender a la patria, por eso como niños ustedes deben aprender la historia de su país; mientras esto sucedía en otras partes también sucedían cosas como por ejemplo la Revolución Rusa"

Elaborar, esta forma de enseñar se presenta en la práctica docente de las cinco profesoras, puesto que la acción que se realiza es que los alumnos leen in trabajo que elaboraron, que generalmente es un cuestionario o un escrito breve acerca de un punto específico del contenido que se esté abordando, la situación es que escriben lo que copiaron del libro de texto, por lo que se manifiesta una ausencia de producción por parte de los alumnos, para sustentar lo anterior se presenta un pasaje de lo acontecido en la observación de clase, en donde se ilustra esta forma de enseñar.

La maestra 1B al tratar de utilizar esta forma de enseñar, parece que no es del interés de los alumnos porque en algunos casos no hicieron nada y en otros parece ser que no entendieron lo que tenían que elaborar que era una noticia, por lo que el contenido queda sin comprenderse, ya que lo que presentan no tiene ninguna relación:

Ma. Manda, a un Ao que ha estado parado, platicando y gritando, a la Dirección. Les dice: "ayer les dejé de tarea que vieran las noticias y cuales son sus partes ¿Cuáles son?"

Aos. Contestan a gritos las partes: "¿Qué?, ¿Quién ¿, ¿Dónde? y ¿Cuándo?"

Ma. Los organiza en equipos y determina que el 1 y el 3, el texto del Plan de San Luis Potosí lo conviertan en noticia, y los equipos 2 y 4 el texto Comienzo de la Revolución, también lo conviertan en noticia. "Les voy a dar 20 minutos para que ló elaboren"

Aos. Continuan, algunos platicando y otros parados sin hacer nada

Ma. Pasa a cada equipo para decirles que realicen el trabajo, preguntándoles: " ¿Cómo van?, ¿Ya mero terminan?" y recordándoles que el tiempo que les queda para terminar.

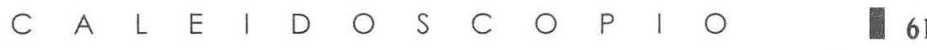


Ma. Les dice: " los que tienen su noticia la van a leer, empezamos porque ya se pasaron del tiempo" (la mayor parte de los Aos. están de pie y platicando) y les reconviene: " el equipo que interrumpa la presentación de sus compañeros los dejaré hasta después de la una de la tarde"

Eq. Pasan los Aos. y uno de ellos lee lo que hicieron (el trabajo que presentan no es noticia sino una entrevista)

Ma. Le dice: "no fue una noticia sino una entrevista" . Continua la presentación con el siguiente equipo.

Ao. Lee su noticia, (no hay ningún comentario, ya que la mayoría de los Aos. platican y comienzan a aventarse bolas de papel y plumas)

Ma. Sin hacer caso a lo que está sucediendo, sólo les dice: "vamos a escuchar al siguiente equipo". Agregando: "voy a contar hasta tres para que se callen y todos en su lugar". Algunos Aos. se callan, otros siguen platicando.

Ma. "insisto vamos a poner atención a Alejandra para irnos"

Eq. Un equipo presenta una escenificación con la noticia que elaboraron (varios

Aos. continuan platicando)

Ma. Interrumpe la escenificación, para callar al grupo y no permite que continue la participación del equipo y vuelve a reiterar que se callen y que se ubiquen en su lugar, algunos de los Aos. le hacen caso pero la mayoría no.

Eq. Otro equipo presenta su noticia

Ma. No permite que continúe el equipo y determina que los Aos. salgan, puesto que ya es hora. Concluye la sesión.

Estos pasajes de las observaciones en el aula de las diferentes maestras, nos muestra que se utilizan diversas formas de enseñar, las cuales están matizadas por las concepciones e importancia que le otorgan a la asignatura de Historia, por ello se aplican con una intencionalidad diferente a lo que establece el constructivismo, en consecuencia la Historia que se enseña, no propicia los niveles conceptuales de cronología, simultaneidad y continuidad, necesarios para construir y desarrollar la noción de tiempo histórico, que como ya se ha planteado es esencial para el aprendizaje de esta asignatura y la formación de un sujeto con un pensamiento histórico que le permita modificar su realidad concreta.

NIVELES DE CONCEPTUALIZACIÓN DEL TIEMPO HISTÓRICO DE LOS ALUMNOS DE 6 GRADO DE EDUCACIÓN PRIMARIA

Como lo plantea la SEP24 para los niños es muy difícil tener un concepto de lo que es el tiempo histórico, ya que la idea de pasado se refiere a lapsos breves y lo relaciona con sus experiencias y con su familia; de 
ahí la dificultad para que los alumnos de educación primaria se formen la imagen de lo que existía antes y la medición del tiempo (lustros, décadas, siglos, etc.).

Por lo cual es importante atender la construcción de ciertas nociones (tiempo histórico, espacialidad, causalidad, movimiento, continuidad y cambio) en el alumno, con el propósito de favorecer el desarrollo de ellas, para que de esta manera puedan comprender los hechos históricos así como su ubicación tanto en el espacio como en el tiempo.

Pozo y Carretero ${ }^{25}$, afirman que el tiempo histórico (TH) con frecuencia es una preocupación prioritaria en todas las discusiones sobre el aprendizaje y enseñanza de la historia ya que tiene diversos componentes, porque se refiere a diferentes fenómenos, una clasificación tentativa es la siguiente:

- Nociones de duración, simultaneidad y sucesión. A este respecto se puede decir que los hechos históricos duran más o menos tiempo y se relacionan entre sí, el instrumento utilizado para su medición es la cronología, por medio de la cual se establecen eras, épocas y períodos históricos, en donde se representan a través de medios matemáticos; los niños y adolescentes suelen confundir a la cronología con el tiempo que ésta mide, sin comprender que un mismo tiempo se puede medir de diversas formas (era cristiana, era musulmana), de lo que se deduce que el carácter conceptual y no de los hechos de la cronología es ignorado totalmente. De ahí que los alumnos tengan serias dificultades para realizar simples problemas matemáticos con fechas históricas, reflejándose en la incomprensión de las fechas históricas.

- Nociones de sucesión o seriación causal histórica. En su entorno inmediato el niño sabe que las causas preceden a los efectos, sin embargo esta comprensión se dificulta y se retrasa en gran medida en los hechos

\footnotetext{
Op. cit.

J. L. Pozo y M. Carretro, "Como enseñar el pasado para entnder el presente" en Sociedad y trabajo de los sujetos en el proceso de enseñanza y aprendizaje. Anexo, México, UPN/SEP, 1988, pp. 414-423.
} 
históricos, lo que pareciera ser se debe, al problema de establecer una causa o un efecto en la historia. La realización de series causales exige "situar la motivación antes de la acción y ordenar dos consecuencias en términos de efectos inmediatos y remotos." 26

- Continuidad pasado - presente - futuro. El establecimiento de efectos remotos se logra comprender cuando el presente es consecuencia directa del pasado y será la causa del futuro, es una de las nociones que se desarrolla evolutivamente en el sujeto más tardíamente y de manera más compleja.

$\operatorname{Jurd}^{27}$, plantea que el concepto de $\mathrm{TH}$, se puede desagregar, dependiendo de las operaciones intelectuales que se precisen, estableciendo por consiguiente tres niveles de conceptualización que son el referente para esta investigación:

- El orden de acontecimientos dentro de una secuencia (Cronología).

- El agrupamiento de acontecimientos que concurren en el tiempo (Simultaneidad).

- El establecimiento de una relación entre el pasado y el presente (Continuidad).

Con los resultados obtenidos se pueden establecer los niveles de conceptualización de la noción de tiempo histórico (TH) de los alumnos estudiados, por escuela y por estrato socioeconómico, tal como se presentan en el cuadro $\mathrm{N}^{\circ} 3$ :

CuAdro 3. Niveles de CONCEPTUALIZACión DE TH POR ESCUELA

\begin{tabular}{|c|c|c|c|}
\hline ESCUELA & $\begin{array}{r}\text { NIVELES } \\
\text { CRONOLOGÍA }\end{array}$ & $\begin{array}{l}\text { CONCEPTUALIZACIÓ } \\
\text { SIMULTANEIDAD }\end{array}$ & $\begin{array}{l}\text { DEL TH } \\
\text { CONTINUIDAD }\end{array}$ \\
\hline $\begin{array}{l}\text { Escuela } 1 \\
\text { (Estrato socioeconómico } \\
\text { ALTO) }\end{array}$ & MEDIO & MEDIO & BAIO \\
\hline $\begin{array}{l}\text { Escuela } 2 \\
\text { (Estrato socioeconómico } \\
\text { MEDIO) }\end{array}$ & MEDIO & BAJO & BAIO \\
\hline $\begin{array}{l}\text { Escuela } 3 \\
\text { (Estrato socioeconómico } \\
\text { BAJO) }\end{array}$ & MEDIO & BAJO & BAJO \\
\hline
\end{tabular}

26 Ibidem., p. 419

27 En Carretero, Asensio y Pozo, op. cit. 
Se puede apreciar que los alumnos de las tres escuelas tienen elementos para lograr lo que establece el nivel de conceptualización de ordenamientos cronológicos de un suceso, ya que en todos los casos se ubican en el nivel medio, lo que significa que por lo menos existe en estos alumnos la capacidad para hacer ordenamientos cronológicos, concepto importante para la comprensión de los contenidos de Historia, entonces quiere decir que se ha construido esta base conceptual para el aprendizaje de esta asignatura.

En cuanto al nivel de conceptualización de simultaneidad, es decir el agrupamiento de sucesos en un tiempo determinado, salvo en el caso de la escuela 1 (estrato socioeconómico alto) en la que los alumnos si tienen los elementos conceptuales para realizar este tipo de agrupamiento ya que se encuentran en un nivel medio, lo que les permite tener mejores elementos para lograr el aprendizaje de los contenidos de Historia; en cambio en las escuelas 2 y 3 los resultados muestran que se encuentran en un nivel bajo, por lo que se considera que no son capaces para realizar este proceso, por consiguiente existen problemas para lograr el aprendizaje de los contenidos de esta asignatura.

En el nivel de conceptualización de relación presente - pasado (continuidad), en los resultados se evidencia que en ninguna de las escuelas, los alumnos cuentan con los elementos necesarios para construir este concepto, ya que se ubican en el nivel bajo, lo que implica que existen serias dificultades para que los alumnos en el proceso de aprendizaje de los contenidos de Historia logren lo que se propone en el programa de esta asignatura que es el de propiciar la conciencia histórica a partir del análisis de la situación actual del país y del mundo como producto del pasado.

El TH es una noción que el alumno construye a partir de los niveles conceptuales de cronología, simultaneidad y de continuidad, como base para explicarse la causalidad de los hechos y su relación con la temporalidad; por ello es que la noción de TH es un sistema de construcción que el sujeto realiza conjuntamente con las otras nociones temporales. Por lo que un aspecto principal que se debe de señalar es el carácter constructivo del aprendizaje de la noción de TH, como lo afirman Carretero, Pozo y Asensio (1989), considerando que para ello el sujeto utiliza muchos elementos y nociones temporales ya construi-

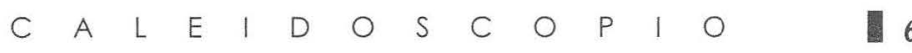


das (tiempo personal, tiempo físico, tiempo social o convencional) e integradas a su propio conocimiento.

\section{REFLEXIONES FINALES}

La enseñanza y el aprendizaje son dos procesos imbricados en la acción educativa, situación que no es una novedad, pero lo que si podemos considerar como relevante para el caso de esta investigación es que implicada en la enseñanza que realizan las profesoras se encuentra su trabajo docente matizado por una serie de elementos que se encuentran asociados y que en este trabajo se describen algunos de ellos como son la concepción que tienen sobre la disciplina de Historia, derivado de ésta como recuperan el enfoque formativo de esta asignatura para los alumnos de educación primaria y con todo este bagaje describir las formas de enseñar utilizadas en esta asignatura.

Con los resultados obtenidos en relación a los elementos asociados al trabajo docente de las profesoras, primero en cuanto a la concepción de la Historia en las cinco maestras del estudio permea la idea de que esta disciplina se encarga del estudio de los hechos del pasado, que en nada ayuda para concebir una historia viva que propicie en los alumnos una formación como sujetos que hacen historia; referente al otro elemento asociado el de la importancia que tiene para ellas esta asignatura en la formación del alumno, aunque le asignan una relativa importancia formativa, se enfoca sobre todo a los valores patrios y a la identidad nacional, la cual se explica con la concepción que poseen sobre esta disciplina, que se traduce en una ausencia formativa para los alumnos de primaria, en términos de generar un pensamiento histórico, que les permita asumir una actitud crítica sobre los acontecimientos sucedidos que los lleve a comprender la situación actual y a tomar conciencia de la posibilidad que existe de cambiarla en beneficio de la colectividad.

Por último las formas de enseñar utilizadas por las docentes (lectura mecánica, cuestionarios y la memorización), parece ser que se ven influidas por los otros dos elementos, ya que los resultados obtenidos nos muestran que casi todas usan las mismas formas, a excepción de dos de ellas que utilizan una más, pero lo que llama la atención es que las tres formas comunes son leer con los alumnos, ejercitar y repetir y 
elaborar, lo que se debe tomar en cuenta en este caso es que en estas formas el sujeto protagónico es la maestra y no el alumno, es decir, que todo el proceso es dirigido por la docente, lo que poco contribuye para desarrollar un aprendizaje constructivo en los alumnos y mucho menos para desarrollar la noción de TH en el caso de la enseñanza de la Historia, tal y como lo muestran los resultados obtenidos en los niveles de conceptualización alcanzados por los grupos atendidos por las profesoras investigadas.

El desarrollo de los niveles de conceptualización del TH en los alumnos se ven poco favorecidos por los docentes, lo que genera serias dificultades para aprender los contenidos de Historia.

En síntesis los datos que se describen en este trabajo plantean que al parecer el desempeño docente respecto a las cinco profesoras de $6^{\circ}$ grado de educación primaria de tres escuelas de diferentes estratos socioeconómicos de la ciudad de Aguascalientes, no son suficientes para desarrollar en los alumnos la noción de TH, la cual conjuntamente con otras, son necesarias para que el alumno logre el aprendizaje de los contenidos de esta asignatura.

Este trabajo sólo describe lo que realizan un grupo muy reducido de docentes con respecto a la forma de propiciar una de las nociones indispensables para que los alumnos aprendan Historia, por lo que queda pendiente el proponer estrategias didácticas que permitan el desarrollo de esas nociones así como el proceso que realizan los alumnos para desarrollarlas, que se pueden convertir en líneas de investigación.

* 
